\title{
Particle Filtering and Cramer-Rao Lower Bound for Underwater Navigation
}

\author{
Rickard Karlsson, Fredrik Gustafsson \\ Tobias Karlsson \\ Division of Communication Systems \\ Department of Electrical Engineering \\ Linköpings universitet, SE-581 83 Linköping, Sweden \\ WWW: http://Www. control.isy.liu.se \\ E-mail: rickard@isy.liu.se, fredrik@isy.liu.se
}

28th October, 2002

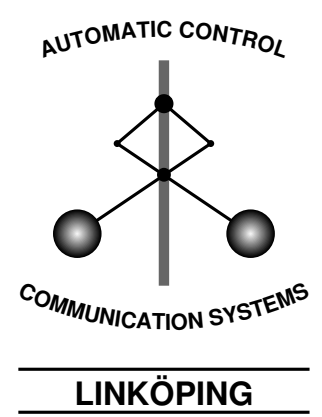

Report no.: LiTH-ISY-R-2474

Submitted to ICASSP 2003

Technical reports from the Control \& Communication group in Linköping are available at http://www.control.isy.liu.se/publications. 


\begin{abstract}
We have studied a sea navigation method relying on a digital underwater terrain map and sonar measurements. The method is applicable for both ships and underwater vessels. We have used experimental data to build an underwater map and to investigate the estimation performance. Since the problem is non-linear, due to the measurement relation, we apply a sequential Monte Carlo method, or particle filter, for the state estimation. The fundamental limitations in navigation uncertainty can be described in terms of the Cramér-Rao lower bound, which is interpreted in terms of the inertial navigation system (INS) error, the sensor accuracy and the terrain map excitation. Hence, the Cramér-Rao lower bound can be interpreted and used in design for INS systems, sensor performance or if these are given, how much terrain or depth excitation that is needed for use in positioning and navigation.
\end{abstract}

Keywords: Particle filter, Cramer-Rao lower bound, Underwater Navigation 


\section{PARTICLE FILTERING AND CRAMÉR-RAO LOWER BOUND FOR UNDERWATER NAVIGATION}

Rickard Karlsson, Fredrik Gustafsson

\author{
Dept. of Electrical Engineering \\ Linköping University \\ SE-581 83 Linköping, Sweden \\ E-mail: $\{$ rickard, fredrik\}@isy.liu.se
}

\author{
Tobias Karlsson \\ Saab Bofors Underwater Systems \\ Box 910 \\ SE-59129 Motala, Sweden \\ E-mail: tobias.karlsson.lithedynamics.saab.se
}

\begin{abstract}
We have studied a sea navigation method relying on a digital underwater terrain map and sonar measurements. The method is applicable for both ships and underwater vessels. We have used experimental data to build an underwater map and to investigate the estimation performance. Since the problem is non-linear, due to the measurement relation, we apply a sequential Monte Carlo method, or particle filter, for the state estimation. The fundamental limitations in navigation uncertainty can be described in terms of the CramérRao lower bound, which is interpreted in terms of the inertial navigation system (INS) error, the sensor accuracy and the terrain map excitation. Hence, the Cramér-Rao lower bound can be interpreted and used in design for INS systems, sensor performance or if these are given, how much terrain or depth excitation that is needed for use in positioning and navigation.
\end{abstract}

\section{INTRODUCTION}

The primary navigation system is in many applications the INS. However, for most applications these systems suffer from small drift errors and must be supported by some external system to correct the errors. This can be done by incorporation GPS measurements. In some applications this is not possible, since the system can not rely on GPS information or these signals can not be received. For underwater navigation we propose a navigation method based on terrain navigation similar to the aircraft terrain navigation proposed in [1]. In [2], several different positioning and navigation systems for particle filtering are discuessed. Here we use an underwater map to support our navigation system. Since the depth information is highly non-linear we use the particle filter method for state estimation. By investigating the Cramér-Rao lower bound from [1,3] we can decide the sensor accuracy needed or if the map yield sufficient information for navigation.

In Fig. 1, the underwater navigation system is described.
The sonar measurement is denoted $s_{t}$, the underwater vessel's depth, $d_{t}$, and the depth from the terrain database in the location $x_{t}$ is denoted $h\left(x_{t}\right)$.

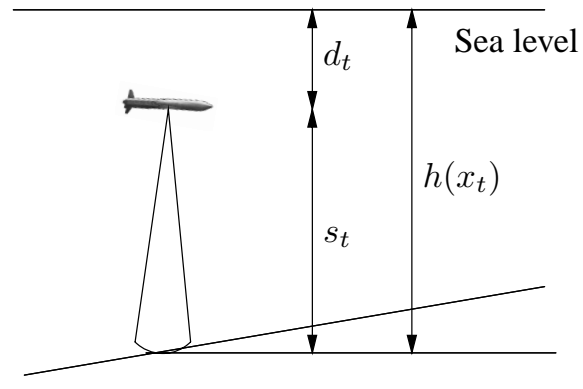

Fig. 1. Underwater navigation using depth information.

\section{PARTICLE FILTER}

Navigation problems are often treated as Bayesian interference. Underwater navigation using a depth map to support the INS is a non-linear problem, therefore the underlying Bayesian equations are non-tractable. To solve in an online application without using linearization or Gaussian assumptions, sequential Monte Carlo methods, or particle filters, could be used. The simulation based ideas have been discussed in [4], where the conditional mean and covariance were calculated using importance sampling for recursive Bayesian estimation. However, the crucial resampling step introduced in $[5,6]$ solved the divergence problems. In this section the presentation of the particle filter theory is according to $[1,3,6,7]$.

Consider the following non-linear discrete-time system

$$
\begin{aligned}
x_{t+1} & =f\left(x_{t}, v_{t}\right) \\
y_{t} & =s_{t}+d_{t}=h\left(x_{t}, e_{t}\right),
\end{aligned}
$$

where $x_{t} \in \mathbb{R}^{n}$ denotes the state of the system and where $y_{t}$ is the observation at time $t$. The process noise $v_{t}$ and mea- 
surement noise $e_{t}$ are assumed independent with densities $p_{v_{t}}$ and $p_{e_{t}}$ respectively. The particle filter method provides an approximative Bayesian solution to discrete-time recursive problem by updating an approximative description of the posterior filtering density. Let $\mathbb{Y}_{t}=\left\{y_{i}\right\}_{i=1}^{t}$ be the set of observations until present time. The Monte Carlo filter approximates the probability density $p\left(x_{t} \mid \mathbb{Y}_{t}\right)$ by a large set of $N$ particles $\left\{x_{t}^{(i)}\right\}_{i=1}^{N}$, where each particle has an assigned relative weight, $w_{t}^{(i)}$, such that all weights sum to unity. The location and weight of each particle reflect the value of the density in the region of the state space. The particle filter updates the particle location and the corresponding weights recursively with each new observation. The non-linear prediction density $p\left(x_{t} \mid \mathbb{Y}_{t-1}\right)$ and filtering density $p\left(x_{t} \mid \mathbb{Y}_{t}\right)$ for the Bayesian interference, [8], are given by

$$
\begin{aligned}
p\left(x_{t+1} \mid \mathbb{Y}_{t}\right) & =\int_{\mathbb{R}^{n}} p\left(x_{t+1} \mid x_{t}\right) p\left(x_{t} \mid \mathbb{Y}_{t}\right) d x_{t} \\
p\left(x_{t} \mid \mathbb{Y}_{t}\right) & =\frac{p\left(y_{t} \mid x_{t}\right) p\left(x_{t} \mid \mathbb{Y}_{t-1}\right)}{p\left(y_{t} \mid \mathbb{Y}_{t-1}\right)}
\end{aligned}
$$

The likelihood $p\left(y_{t} \mid x_{t}\right)$ is calculated from (1) using the known measurement noise density $p_{e_{t}}$. An often used assumption is additive noise, so $y_{t}=h\left(x_{t}\right)+e_{t}$, yielding

$$
w_{t}=p\left(y_{t} \mid x_{t}\right)=p_{e_{t}}\left(y_{t}-h\left(x_{t}\right)\right) .
$$

The main idea is to approximate $p\left(x_{t} \mid \mathbb{Y}_{t-1}\right)$ with a sum of delta-Dirac functions located in the samples, $x_{t}^{(i)}$. Using the importance weights the posterior can be written as

$$
p\left(x_{t} \mid \mathbb{Y}_{t}\right) \approx \sum_{i=1}^{N} \tilde{w}_{t}^{(i)} \delta\left(x_{t}-x_{t}^{(i)}\right)
$$

where the normalized importance weights are defined as

$$
\tilde{w}_{t}^{(i)}=\frac{w_{t}^{(i)}}{\sum_{j=1}^{N} w_{t}^{(j)}}, i=1, \ldots, N
$$

and where $w_{t}^{(i)} \propto p\left(y_{t} \mid x_{t}^{(i)}\right)$. This was the original estimation idea. However, this approach leads to divergence, where almost all of the particles have zero weights. By introducing a selection or resampling step as proposed in [6] this can be handled. This resampling idea from [6] is often referred to as sampling importance resampling (SIR), and the idea is summarized in $\mathrm{Alg} 1$.

\section{Sampling Importance Resampling (SIR)}

1. Set $t=0$ and generate $N$ samples $\left\{x_{0}^{(i)}\right\}_{i=1}^{N}$ from the initial distribution $p\left(x_{0}\right)$.

2. Compute $w_{t}^{(i)}=p\left(y_{t} \mid x_{t}^{(i)}\right)$ and normalize, i.e., $\tilde{w}_{t}^{(i)}=w_{t}^{(i)} / \sum_{j=1}^{N} w_{t}^{(j)}, i=1, \ldots, N$.

3. Generate a new set $\left\{x_{t}^{(i \star)}\right\}_{i=1}^{N}$ by resampling with replacement $N$ times from $\left\{x_{t}^{(i)}\right\}_{i=1}^{N}$, with probability $\tilde{w}_{t}^{(j)}=\operatorname{Pr}\left\{x_{t}^{(i \star)}=x_{t}^{(j)}\right\}$.

4. Predict (simulate) new particles, i.e., $x_{t+1}^{(i)}=$ $f\left(x_{t}^{(i \star)}, v_{t}^{(i)}\right), i=1, \ldots, N$ using different noise realizations for the particles.

5. Increase $t$ and iterate to step 2 .

Alg. 1. Sampling Importance Resampling.

\section{THE CRAMÉR-RAO LOWER BOUND}

We consider the following model for an inertial navigation system (INS) according to [1]

$$
\begin{aligned}
x_{t+1} & =x_{t}+u_{t}+v_{t} \\
y_{t} & =h\left(x_{t}\right)+e_{t},
\end{aligned}
$$

where $x_{t} \in \mathbb{R}^{2}$ is the horizontal position state vector, $u_{t}$ is the INS corrections and $v_{t}$ the process noise due to INS drift. The observation relation consists of sonar measurements of the depth, where $e_{t}$ is the measurement noise. Using standard notations we consider independent noise sources, with variances $Q_{t}=E\left\{v_{t} v_{t}^{T}\right\}$ and $R_{t}=E\left\{e_{t} e_{t}^{T}\right\}$.

The Cramér-Rao lower bound for one step prediction with models according to (6) is given in $[1,3]$. We can formulate this as

$$
P_{t+1}=\left(P_{t}^{-1}+E\left\{\varphi\left(x_{t}\right) R_{t}^{-1} \varphi^{T}\left(x_{t}\right)\right\}\right)^{-1}+Q_{t},
$$

where $P_{t}$ is the covariance matrix for the estimation error, when we evaluate locally around the position $x_{t}$. In (7) we have defined

$$
\varphi\left(x_{t}\right)=\left.\nabla_{x} h(x)\right|_{x=x_{t}} .
$$

For a diagonal measurement noise matrix $R_{t}$, we have

$$
P_{t+1}=\left(P_{t}^{-1}+R_{t}^{-1} Z_{t}\right)^{-1}+Q_{t}
$$

using the definition

$$
Z_{t}=E\left\{\varphi\left(x_{t}\right) \varphi^{T}\left(x_{t}\right)\right\}
$$

We are interested in the stationary behavior in each position, i.e., $Z_{t}=Z(x)$. The assumption is that we get the 
global behavior by studying the covariance locally in each position. For stationary systems, $P_{t} \rightarrow \bar{P}(x), t \rightarrow \infty$, this can be written as

$$
\begin{aligned}
\bar{P}(x) & =\left(\bar{P}^{-1}(x)+R^{-1} Z(x)\right)^{-1}+Q \\
& =\bar{P}(x)\left(I+\bar{P}(x) R^{-1} Z(x)\right)^{-1}+Q \\
& \approx \bar{P}(x)\left(I-\bar{P}(x) R^{-1} Z(x)\right)+Q .
\end{aligned}
$$

Hence the stationary covariance for the Cramér-Rao lower bound is given by (12)

$$
\bar{P}^{2}(x)=Q Z^{-1}(x) R,
$$

under the assumption that the Taylor expansion is valid, $\bar{P}(x) \ll R Z^{-1}(x)$ (in some norm). We can directly interpret this relation, for example increased INS uncertainty yields higher covariance. Conversely, a better sensor (lower $R$ ) or higher terrain excitation (larger $Z$ ) reduces $\bar{P}$. For an important special case, scalar values $r, q$ and independent coordinates in (10) we have

$$
R=r, Q=q I_{2}, Z=\left(\begin{array}{cc}
z_{1} & 0 \\
0 & z_{2}
\end{array}\right) \Rightarrow \bar{P}=\left(\begin{array}{cc}
p_{1} & 0 \\
0 & p_{2}
\end{array}\right),
$$

where $I_{2}$ is the $2 \times 2$ identity matrix. Hence, the system can by solved exactly by studying the scalar equation

$\bar{p}_{i}=\frac{1}{\bar{p}_{i}^{-1}+z_{i} / r}+q \Rightarrow \bar{p}_{i}=\frac{q}{2}+\sqrt{q^{2} / 4+q r / z_{i}}, i=1,2$.

\section{SIMULATIONS}

In this section we implement and evaluate the particle filter on experimental data from an underwater vessel system. We also apply the Cramér-Rao lower bound calculations from Section 3.

In [9] a terrain map for underwater systems was collected using sonar depth measurements and differential GPS. This data is here resampled to a uniform grid and used for navigation and calculation of the Cramér-Rao lower bound . The terrain underwater map is shown in Fig. 2 and in Fig. 5 the data used in the depth interpolation is shown, together with the vessels true trajectory.

The Cramér-Rao lower bound values for each position in the map are given in Fig. 3 for $\log (\|\bar{P}\|)$ according to (12). If the Taylor approximation is not valid, the covariance is iterated until convergence. The expected mean in (10) is implemented as a mean value over different numerical differentiation approximations, e.g Euler-forward, backward etc.

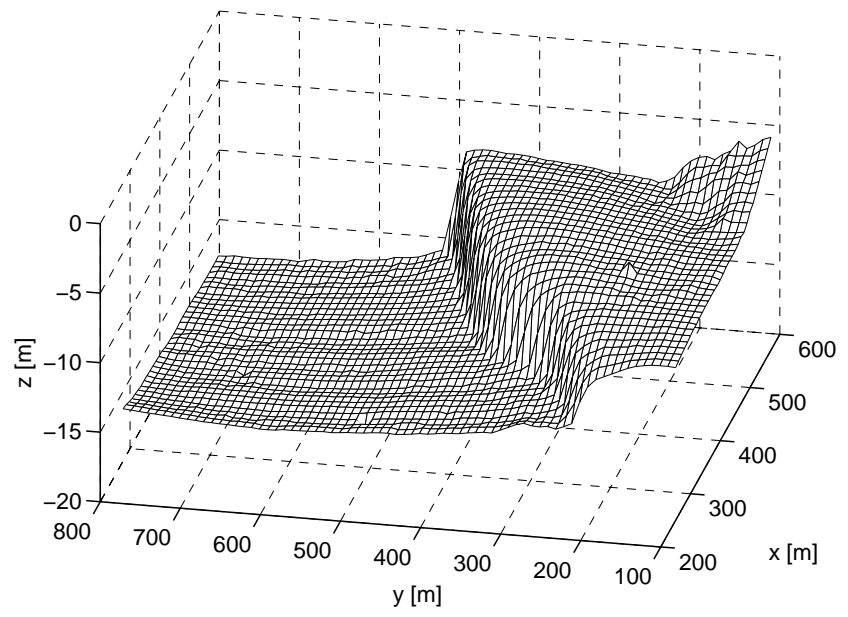

Fig. 2. Underwater terrain information depth map.

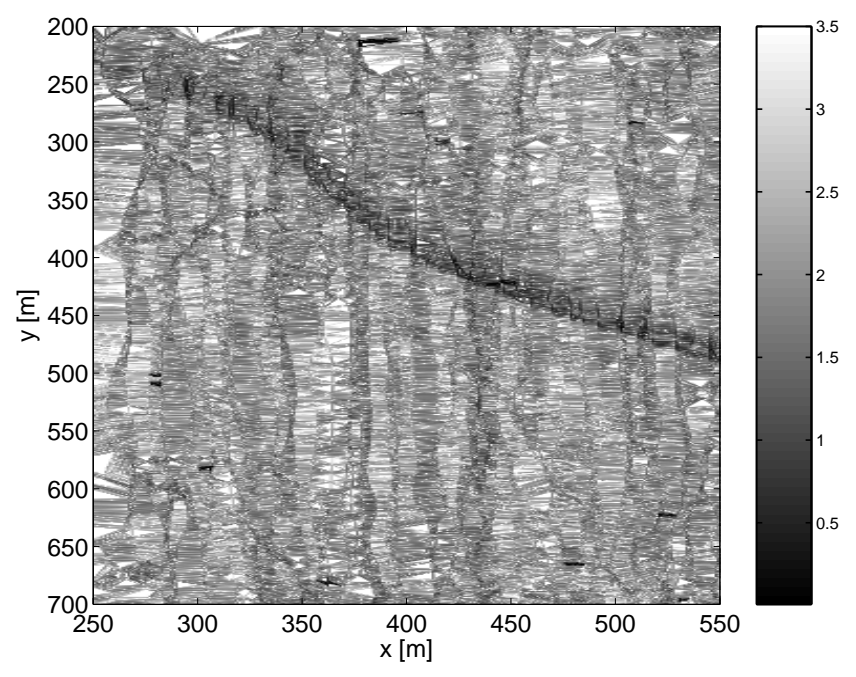

Fig. 3. CRLB presented as $\log (\bar{P})$ in map coordinates.

A particle filter method is then tested on the underwater map. It is initialized be placing particles uniformly over the entire map. The process and measurement noise are assumed Gaussian with covariances $Q=I_{2}$ and $R=0.1$ respectively, but other distributions could easily be used. The particle filter is initialized with $N=20000$ particles, but after a few iteration it is reduced to 5000. The depth of the vessel is considered constant during the simulation. The input signal $u_{t}$ is from the GPS estimate since no true speedometer was present. However, the signal is perturbed to emulate true performance by adding an error of 10 percent with a uniform distribution.

In Fig. 4 the mean value estimate is shown from the particle filter, when a large initial uncertainty is considered (as large as the map). The original sample rate was 10 [Hz], but data was decimated so the filter was updated every 5 [s]. As 
seen, the filter estimate is close to the true position. In Fig. 6 the root mean square error (RMSE) is presented.

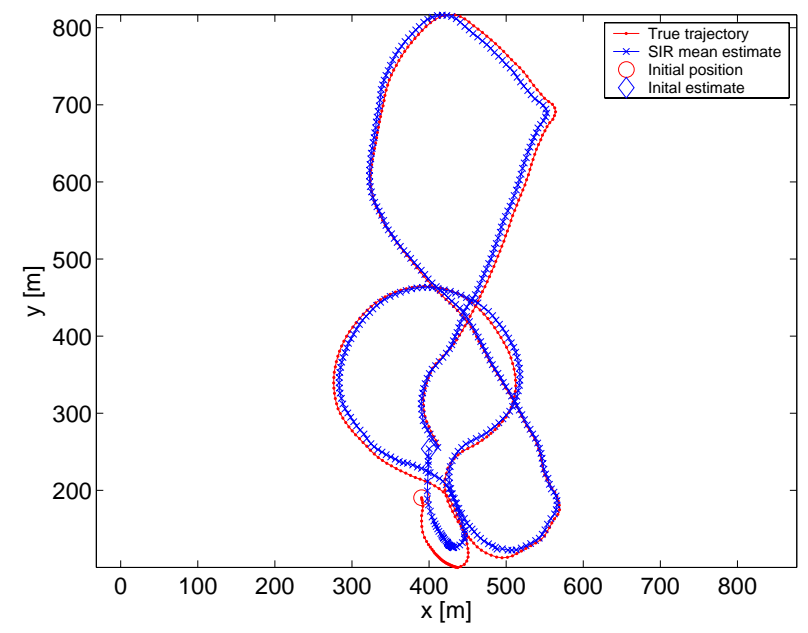

Fig. 4. The mean estimate from the particle filter.

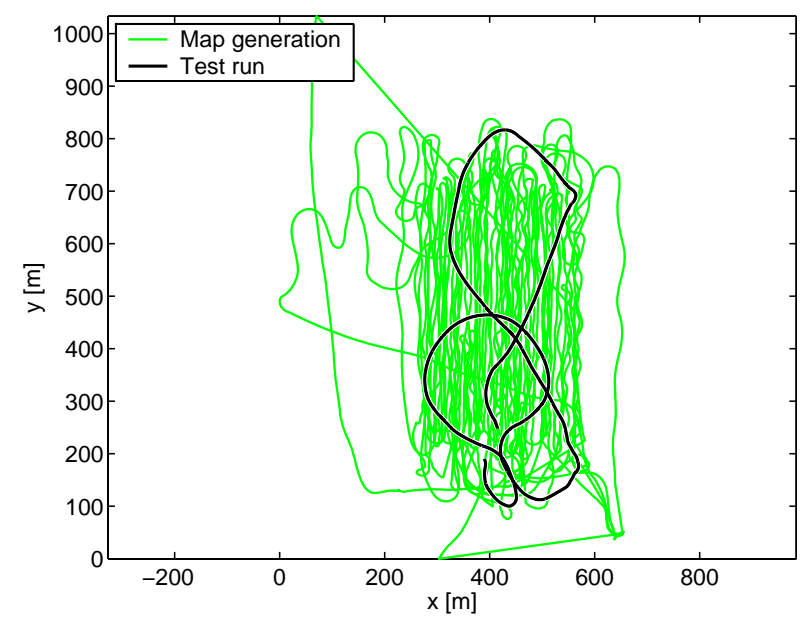

Fig. 5. Underwater vessel trajectory and map generation.

\section{CONCLUSIONS}

In this paper we have constructed a digital underwater map from experimental data, for usage in an underwater navigation application. We have investigated the Cramér-Rao lower bound for the underwater application presented in Section 3, and interpreted it in terms of the INS error, the sensor accuracy and the terrain map excitation. Hence, CramérRao lower bound can be interpreted and used in design for INS systems, sensor performance or if these are given, how much terrain or depth excitation that is needed for use in positioning and navigation using the particle filter. A successful implementation of the particle filter for underwater navigation using the collected depth map was also given, where the presented RMSE gives the performance.

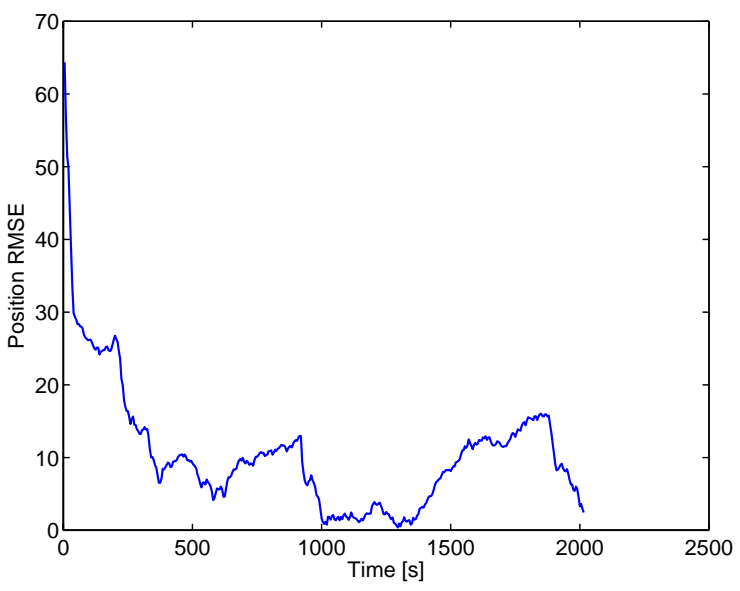

Fig. 6. Root Mean Square Error for the particle filter.

The authors would like to thank Saab Bofors Underwater Systems, Björn Johansson and Anna Falkenberg for providing underwater topological data.

\section{REFERENCES}

[1] N. Bergman, Recursive Bayesian Estimation: Navigation and Tracking Applications, Ph.D. thesis, Linköping University, 1999, Dissertations No. 579.

[2] F. Gustafsson, F. Gunnarsson, N. Bergman, U. Forssell, J. Jansson, R. Karlsson, and P-J Nordlund, "Particle Filters for Positioning, Navigation and Tracking," IEEE Transactions on Signal Processing, Feb 2002, (Feb, 2002).

[3] A. Doucet, N. de Freitas, and N. Gordon, Eds., Sequential Monte Carlo Methods in Practice, Springer Verlag, 2001.

[4] J.E Handschin, "Monte Carlo Techniques for Prediction and Filtering of Non-linear Stochastic Processes," Automatica, vol. 6, pp. 555-563, 1970.

[5] A.F.M Smith and A.E. Gelfand, "Bayesian Statistics Without Tears: A Sampling-Resampling Perspective," The American Statistican, vol. 46, no. 2, pp. 84-88, 1992.

[6] N.J. Gordon, D.J. Salmond, and A.F.M. Smith, "A Novel Approach to Nonlinear/non-Gaussian Bayesian State Estimation," in IEE Proceedings on Radar and Signal Processing, 1993, vol. 140, pp. 107-113.

[7] R. Karlsson, Simulation Based Methods for Target Tracking, Licentiate Thesis no. 930, Department of Electrical Engineering, Linköping University, Sweden, Feb 2002.

[8] A.H. Jazwinski, Stochastic Processes and Filtering Theory, vol. 64 of Mathematics in Science and Engineering, Academic Press, 1970.

[9] T. Karlsson, "Terrain Aided Underwater Navigation using Bayesian Statistics," Masters Thesis LiTH-ISY-EX-3292, Department of Electrical Engineering, Linköping University, 2003, To be published. 